\title{
A radiation condition for uniqueness in a wave propagation problem for 2-D open waveguides
}

\author{
Giulio Ciraolo ${ }^{1, *}$ and Rolando Magnanini ${ }^{2}$ \\ ${ }^{1}$ Dipartimento di Matematica e Applicazioni per l'Architettura, Università di Firenze, Piazza Ghiberti 27, \\ 50122 Firenze, Italy \\ ${ }^{2}$ Dipartimento di Matematica 'U. Dini', Università di Firenze, Viale Morgagni 67/A, 50134 Firenze, Italy
}

Communicated by Y. S. Xu

\section{SUMMARY}

We study the uniqueness of solutions of Helmholtz equation for a problem that concerns wave propagation in waveguides. The classical radiation condition does not apply to our problem because the inhomogeneity of the index of refraction extends to infinity in one direction. Also, because of the presence of a waveguide, some waves propagate in one direction with different propagation constants and without decaying in amplitude.

We provide an explicit condition for uniqueness for rectilinear waveguides, which takes into account the physically significant components, corresponding to guided and non-guided waves; this condition reduces to the classical Sommerfeld-Rellich condition in the relevant cases.

By a careful asymptotic analysis we prove that the solution derived by Magnanini and Santosa (SIAM J. Appl. Math. 2001; 61:1237-1252) for stratified media satisfies our radiation condition. Copyright (C) 2008 John Wiley \& Sons, Ltd.

KEY WORDS: electromagnetic fields; wave propagation; Helmholtz equation; optical waveguides; uniqueness of solutions; radiation condition

\section{THE PROBLEM OF UNIQUENESS FOR THE HELMHOLTZ EQUATION}

Let $\Sigma \subset \mathbb{R}^{N}$ be a (possibly empty) bounded closed surface. It is well known that the Dirichlet problem

$$
\begin{cases}\Delta u+k^{2} u=f & \text { outside } \Sigma \\ u=U & \text { on } \Sigma\end{cases}
$$

\footnotetext{
*Correspondence to: Giulio Ciraolo, Dipartimento di Matematica e Applicazioni per l'Architettura, Università di Firenze, Piazza Ghiberti 27, 50122 Firenze, Italy.

†E-mail: ciraolo@math.unifi.it

Contract/grant sponsor: MIUR
} 


\section{G. CIRAOLO AND R. MAGNANINI}

has no unique solution. If $k=0$ (Poisson's equation), in order to obtain the uniqueness, it is required that the solution vanishes at infinity. If $k \neq 0$, that is not sufficient anymore. In fact, there are two different solutions of (1) which vanish at infinity, representing the outward and inward radiation. Hence, an additional (or different) condition at infinity is needed.

The first condition we can add is that

$$
\lim _{R \rightarrow \infty} R^{N-1 / 2}\left(\frac{\partial u}{\partial R}-\mathrm{i} k u\right)=0
$$

uniformly; this is the so-called Sommerfeld's radiation condition. Here, $\partial u / \partial R$ denotes the radial derivative of $u$. The physical meaning of this condition is that there are no sources of energy at infinity. Moreover, it assures that, far from the surface $\Sigma, u$ behaves as a wave generated by a point source.

Stated as in (2) and together with the assumption that $u$ vanishes at infinity, this condition is due to Sommerfeld, see [1,2] (see also [3,4]). The vanishing assumption on $u$ was dropped by Rellich (see [5]), who also proved that (2) can be replaced by the weaker condition

$$
\lim _{R \rightarrow \infty} \int_{\partial B_{R}}\left|\frac{\partial u}{\partial R}-\mathrm{i} k u\right|^{2} \mathrm{~d} \sigma=0
$$

where $B_{R}$ is the ball centered at the origin with radius $R$. In the same paper, Rellich also proved that a radiation condition can also be given in the form

$$
\int_{\mathbb{R}^{N}}\left|\frac{\partial u}{\partial R}-\mathrm{i} k u\right|^{2} \mathrm{~d} x<+\infty
$$

Condition (4) can be considered the starting point for our work, as we are going to explain shortly. Before describing our results, we cite some generalizations of the work of Rellich.

When $n$ is a function which is identically 1 outside a compact set, (3) still guarantees the uniqueness of a solution of

$$
\Delta u+k^{2} n(x)^{2} u=f, \quad x \in \mathbb{R}^{N}
$$

see $[6,7]$ and references therein. Several authors (see, for instance, $[5,8]$ ) studied the case in which $n$ is not constant at infinity, but has an angular dependency, say $n(x) \rightarrow n_{\infty}(x /|x|)$ as $|x| \rightarrow \infty$, and it approaches to the limit with a certain behavior.

In this kind of problems, the limiting absorption principle (LAP) has been widely used to obtain uniqueness, by approximating problems with a complex-valued index of refraction [9-12]. With this method, uniqueness results for evolutionary problems in stratified media are presented in [13-15]. The LAP for stationary problems has been employed, among others, in [11,16-18]. Among these papers, we wish to mention the results in $[17,18]$, where the authors proved the uniqueness of solutions of the Helmholtz equation by means of the limiting absorbtion method and by introducing the radiation condition:

$$
\lim _{R \rightarrow+\infty} \frac{1}{R} \int_{B_{R}}\left|\frac{\partial u}{\partial R}-\mathrm{i} k n_{\infty} u\right|^{2} \mathrm{~d} x=0
$$

Here, the assumptions on $n$ are such that the energy cannot be trapped along any direction, but it radiates toward infinity. That is in accordance with [19], where the authors point out that the 
Sommerfeld radiation condition, since it involves the dimension, is inappropriate for problems admitting a lower-dimensional solution (a plane wave).

The present paper is motivated by the study of wave propagation in optical waveguides. In particular, we shall study the uniqueness of solutions of the 2-D Helmholtz equation

$$
\Delta u+k^{2} n(x)^{2} u=f, \quad(x, z) \in \mathbb{R}^{2}
$$

where $n$ is of the form

$$
n:= \begin{cases}n_{\mathrm{co}}(x), & |x| \leqslant h \\ n_{\mathrm{cl}}, & |x|>h\end{cases}
$$

here $n_{\mathrm{co}}$ is a bounded function and $n_{\mathrm{cl}}$ is a constant; thus, (6) models the index of refraction of a rectilinear open waveguide of width $2 h$ (subscripts $c o$ and $c l$ refer to the core and cladding of the waveguide) (see [20]).

We observe that functions $n$ of type (6) are not considered in the works cited before. In fact, the most important feature of optical waveguides is the presence of waves confined inside the waveguide (guided modes), which are oscillatory and never decaying along the axis of propagation ( $z$-axis). It is easy to show that a pure guided mode supported by the Helmholtz equation does not satisfy the radiation conditions above retrieved (as already pointed out in [19]). Functions $n$ similar to (6) were considered by Jäger and Saitō [21, 22]; however, their assumptions on $n$ do not admit the occurrence of guided modes.

Works dealing with uniqueness for the Helmholtz equation in an optical waveguide setting have appeared in the Russian literature (see [23-26] and references therein). However, the Reichardt condition studied therein deals only with guided modes and does not apply to the total field.

The first result of this paper is Theorem 2.3, where we present a radiation condition that guarantees the uniqueness of a solution of (5) with $n$ given by (6). We prove Theorem 2.3 without using the LAP and prefer a simple argument that is in the spirit of the results of Rellich and their generalizations (see $[5,7]$ and references therein). We observe that, if we suppose that no guided mode is present (this is possible by choosing special parameters in the function $n$ ), our radiation condition reduces to (4).

With an alternative approach, a condition for uniqueness for stratified media in Euclidean three space is presented in [27] (see also [28,29]). There, a criterium of selection of outgoing waves is developed as a generalization of Sommerfeld's uniform radiation condition. Our condition, as Rellich's, is given in an integral form and it appears to nicely handle the behavior of the waves propagating transversally to the waveguide.

Another interesting result of this paper is the proof that the solution of (5) derived in $[30]^{\ddagger}$ actually satisfies our radiation condition. We accomplish this task in Section 3 by making a careful analysis of the asymptotic behavior of Green's function obtained in [30]. The asymptotic formulas derived in Theorem 3.6 have proven to be useful in [31], where locally perturbed waveguides are considered.

In Section 3 we articulate such a proof into three steps: in Section 3.1 we derive a representation of the solution as a contour integral; in Section 3.2 we prove uniform estimates for the non-guided part of the spectrum-based solution; in Section 3.3 we carry out the proof by testing the radiation condition on the guided part and using the asymptotic estimates obtained in Section 3.2.

${ }^{\ddagger}$ We will refer to such a solution as the spectrum-based solution. 


\section{G. CIRAOLO AND R. MAGNANINI}

We wish to observe that the results in the present paper can be easily adapted to prove the uniqueness of a solution for the Pekeris waveguide problem (see [32, 33]).

\section{A RELLICH-TYPE CONDITION AND A UNIQUENESS THEOREM}

In this section we shall state a radiation condition that generalizes (4) and prove our uniqueness result. The key ingredients of our proof are essentially four: (i) if (5) possesses two solutions satisfying our radiation condition, then their difference $w$ must belong to the Sobolev space $H^{2}\left(\mathbb{R}^{2}\right)$; (ii) as a consequence of (i), the Fourier transform of $w$ in the $z$-direction (parallel to the fiber's axis) is square integrable for almost all $x \in \mathbb{R}$ and satisfies an ordinary differential equation in $x$; (iii) the only square integrable solution of such an equation is identically zero; (iv) the proof is then completed by using an appropriate transform theory in the $x$-direction and repeating the arguments in (ii) and (iii).

\subsection{Preliminaries}

We recall the relevant results of [30], which will be useful in the rest of the paper. In [30], the authors assumed that $n$, given by (6), is a non-increasing function; we note that such an assumption can be dropped by using the Titchmarsh theory on eigenfunction expansion (see $[34,35])$ and replaced by the weaker assumption that $n$ is a bounded function.

In [30] Green's function $G$ for (5) is constructed: a solution of (5) is

$$
u(x, z)=\int_{\mathbb{R}^{2}} G(x, z ; \xi, \zeta) f(\xi, \zeta) \mathrm{d} \xi \mathrm{d} \zeta
$$

where

$$
G(x, z ; \xi, \zeta)=\sum_{j \in\{s, a\}} \int_{0}^{+\infty} \frac{\mathrm{e}^{\mathrm{i}|z-\zeta| \sqrt{k^{2} n_{*}^{2}-\lambda}}}{2 \mathrm{i} \sqrt{k^{2} n_{*}^{2}-\lambda}} v_{j}(x, \lambda) v_{j}(\xi, \lambda) \mathrm{d} \rho_{j}(\lambda)
$$

Here

$$
\begin{aligned}
n_{*} & =\max _{\mathbb{R}} n \\
\left\langle\mathrm{~d} \rho_{j}, \eta\right\rangle & =\sum_{m=1}^{M_{j}} r_{m}^{j} \eta\left(\lambda_{m}^{j}\right)+\frac{1}{2 \pi} \int_{d^{2}}^{+\infty} \frac{\sqrt{\lambda-d^{2}}}{\left(\lambda-d^{2}\right) \phi_{j}(h, \lambda)^{2}+\phi_{j}^{\prime}(h, \lambda)^{2}} \eta(\lambda) \mathrm{d} \lambda
\end{aligned}
$$

for all $\eta \in C_{0}^{\infty}(\mathbb{R})(\langle$,$\rangle is the usual dual product )$,

$$
r_{m}^{j}=\left[\int_{-\infty}^{+\infty} v_{j}\left(x, \lambda_{m}^{j}\right)^{2} \mathrm{~d} x\right]^{-1}=\frac{\sqrt{d^{2}-\lambda_{m}^{j}}}{\sqrt{d^{2}-\lambda_{m}^{j}} \int_{-h}^{h} \phi_{j}\left(x, \lambda_{m}^{j}\right)^{2} \mathrm{~d} x+\phi_{j}\left(h, \lambda_{m}^{j}\right)^{2}}
$$

Also, $v_{j}(x, \lambda)$ are linearly independent solutions of

$$
v^{\prime \prime}+[\lambda-q(x)] v=0 \quad \text { in } \mathbb{R}
$$


where $q(x)=k^{2}\left[n_{*}^{2}-n(x)^{2}\right]$ and has the following form:

$$
v_{j}(x, \lambda)= \begin{cases}\phi_{j}(h, \lambda) \cos Q(x-h)+\frac{\phi_{j}^{\prime}(h, \lambda)}{Q} \sin Q(x-h) & \text { if } x>h \\ \phi_{j}(x, \lambda) & \text { if }|x| \leqslant h \\ \phi_{j}(-h, \lambda) \cos Q(x+h)+\frac{\phi_{j}^{\prime}(-h, \lambda)}{Q} \sin Q(x+h) & \text { if } x<-h\end{cases}
$$

for $j=s$, $a$, with $Q=\sqrt{\lambda-d^{2}}, d^{2}=k^{2}\left(n_{*}^{2}-n_{\mathrm{cl}}^{2}\right)$; the $\phi_{j}$ 's are solutions of (10) in the interval $(-h, h)$ and satisfy the initial conditions:

$$
\begin{array}{ll}
\phi_{s}(0, \lambda)=1, & \phi_{s}^{\prime}(0, \lambda)=0 \\
\phi_{a}(0, \lambda)=0, & \phi_{a}^{\prime}(0, \lambda)=\sqrt{\lambda}
\end{array}
$$

(The indices $j=s, a$ correspond to symmetric and antisymmetric solutions, respectively.)

We note that (8) can be split up into two summands:

$$
G=G^{g}+G^{\mathrm{rad}}
$$

where

$$
G^{g}(x, z ; \xi, \zeta)=\sum_{j \in\{s, a\}} \sum_{m=1}^{M_{j}} \frac{\mathrm{e}^{\mathrm{i}|z-\zeta| \sqrt{k^{2} n_{*}^{2}-\lambda_{m}^{j}}}}{2 \mathrm{i} \sqrt{k^{2} n_{*}^{2}-\lambda_{m}^{j}}} v_{j}\left(x, \lambda_{m}^{j}\right) v_{j}\left(\xi, \lambda_{m}^{j}\right) r_{m}^{j}
$$

and

$$
G^{\mathrm{rad}}(x, z ; \xi, \zeta)=\frac{1}{2 \pi} \sum_{j \in\{s, a\}} \int_{d^{2}}^{+\infty} \frac{\mathrm{e}^{\mathrm{i}|z-\zeta| \sqrt{k^{2} n_{*}^{2}-\lambda}}}{2 \mathrm{i} \sqrt{k^{2} n_{*}^{2}-\lambda}} v_{j}(x, \lambda) v_{j}(\xi, \lambda) \frac{\sigma_{j}(\lambda)}{\sqrt{\lambda-d^{2}}} \mathrm{~d} \lambda
$$

with

$$
\sigma_{j}(\lambda)=\frac{\lambda-d^{2}}{\left(\lambda-d^{2}\right) \phi_{j}(h, \lambda)^{2}+\phi_{j}^{\prime}(h, \lambda)^{2}}, \quad j=s, a
$$

$G^{g}$ represents the guided part of Green's function, which involves the guided modes, i.e. the modes propagating mainly inside the waveguide; $G^{\text {rad }}$ is the part of Green's function corresponding to the non-guided energy, i.e. the energy radiated outside or vanishing along the waveguide, which we denote by

$$
u^{\mathrm{rad}}=\int_{\mathbb{R}^{2}} G^{\mathrm{rad}}(x, z ; \xi, \zeta) f(\xi, \zeta)
$$

It exists a finite number of guided modes, which corresponds to the finite number of roots of the equations

$$
\sqrt{d^{2}-\lambda} \phi_{j}(h, \lambda)+\phi_{j}^{\prime}(h, \lambda)=0, \quad j \in\{s, a\}
$$




\section{G. CIRAOLO AND R. MAGNANINI}

laying in the interval $\left(0, d^{2}\right)$. We shall denote by $\lambda_{m}^{j}, m=1, \ldots, M_{j}, j=s, a$, these roots. Each $v_{j}\left(x, \lambda_{m}^{j}\right)$ decays exponentially for $|x|>h$ as it is clear from the formula:

$$
v_{j}\left(x, \lambda_{m}^{j}\right)= \begin{cases}\phi_{j}\left(h, \lambda_{m}^{j}\right) \mathrm{e}^{-\sqrt{d^{2}-\lambda_{m}^{j}}(x-h)}, & x>h \\ \phi_{j}\left(x, \lambda_{m}^{j}\right), & |x| \leqslant h \\ \phi_{j}\left(-h, \lambda_{m}^{j}\right) \mathrm{e}^{\sqrt{d^{2}-\lambda_{m}^{j}}(x+h)}, & x<-h\end{cases}
$$

We note that $G^{g}$ is bounded and oscillatory in the $z$-direction, because $\sqrt{k^{2} n_{*}^{2}-\lambda_{m}^{j}}$ is real for every $m=1, \ldots, M_{j}, j=s, a$.

\section{Remark 2.1}

The functions $\sigma_{j}(\lambda), j \in\{s, a\}$, given by (14), are meromorphic functions of $\lambda \in \mathbb{C}$, real-valued for $\lambda \in \mathbb{R}$ and with poles that are real and simple (see $[34,36])$, which corresponds to the values $\lambda_{m}^{j}$, $m=1, \ldots, M_{j}, j=s, a$.

To simplify notations, we shall denote by $\gamma_{l}, l=1, \ldots, M, M=M_{s}+M_{a}$, the values $\lambda_{m}^{j}, m=$ $1, \ldots, M_{j}, j=s, a$, ordered according to the natural ordering on the real line, and by $\gamma_{*}$ their maximum. With these premises, we set

$$
e\left(x, \gamma_{l}\right)=\frac{v_{j}\left(x, \gamma_{l}\right)}{\left\|v_{j}\left(\cdot, \gamma_{l}\right)\right\|_{L^{2}(\mathbb{R})}}
$$

From (12) and (9), it is clear that the guided part $G^{g}$ can be expressed as

$$
G^{g}(x, z ; \xi, \zeta)=\sum_{l=1}^{M} G_{l}^{g}(x, z ; \xi, \zeta)
$$

where

$$
G_{l}^{g}(x, z ; \xi, \zeta)=\frac{\mathrm{e}^{\mathrm{i} \beta_{l}|z-\zeta|}}{2 \mathrm{i} \beta_{l}} e\left(x, \gamma_{l}\right) e\left(\xi, \gamma_{l}\right)
$$

with

$$
\beta_{l}=\sqrt{k^{2} n_{*}^{2}-\gamma_{l}}
$$

Let $s \in \mathbb{R}$; we will denote by $L^{2, s}\left(\mathbb{R}^{2}\right)$ the weighted Lebesgue space consisting of all the complexvalued measurable functions $u$ such that $\left(1+x^{2}+z^{2}\right)^{s}|u(x, z)|^{2}$ is summable in $\mathbb{R}^{2}$, equipped with the natural norm defined by

$$
|u|_{2, s}^{2}=\int_{\mathbb{R}^{2}}|u(x, z)|^{2}\left(1+x^{2}+z^{2}\right)^{s} \mathrm{~d} x \mathrm{~d} z
$$

$L^{2, s}\left(\mathbb{R}^{2}\right)$ is commonly used when dealing with solutions of Helmholtz equation (see $\left.[37,38]\right)$. In [39] we proved that the spectrum-based solution (7)-(8) derived in [30] belongs to $L^{2, s}\left(\mathbb{R}^{2}\right)$, for $s<-1$, if $f \in L^{2,-s}\left(\mathbb{R}^{2}\right)$.

The following lemma will be useful in the following subsection. 
Lemma 2.2

For $s<-1$, let $u \in L^{2, s}\left(\mathbb{R}^{2}\right)$ satisfy

$$
\Delta u+k^{2} n(x)^{2} u=0
$$

in $\mathbb{R}^{2}$, where $n$ is given by (6). Then

$$
\lim _{|x| \rightarrow+\infty} u(x, z) \mathrm{e}^{-|x| \sqrt{d^{2}-\gamma_{*}}}=\lim _{|x| \rightarrow+\infty} u_{x}(x, z) \mathrm{e}^{-|x| \sqrt{d^{2}-\gamma_{*}}}=0
$$

where $\gamma_{*}=\max _{1 \leqslant l \leqslant M} \gamma_{l}$.

Proof

Since $u$ is a solution of (20), from Lemmas A.1 and A.3 in [39], we infer that both $\left(1+x^{2}+\right.$ $\left.z^{2}\right)^{s}|\nabla u(x, z)|^{2}$ and $\left(1+x^{2}+z^{2}\right)^{s}\left|\nabla^{2} u(x, z)\right|^{2}$ are summable in $\mathbb{R}^{2}$. Thus, it easily follows that the function

$$
\Psi(x, z)=\left(1+x^{2}+z^{2}\right)^{s / 2} u(x, z)
$$

belongs to the Sobolev space $W^{2,2}\left(\mathbb{R}^{2}\right)$. The Sobolev imbedding theorem (see Theorem 4.12 in [40]) implies that $\Psi \in L^{\infty}\left(\mathbb{R}^{2}\right)$ and hence the first limit in (21) follows at once.

A straightforward computation shows that $\Psi$ satisfies the following equation:

$$
\Delta \Psi+b \cdot \nabla \Psi+c \Psi=0
$$

in $\mathbb{R}^{2}$, where

$$
b(x, z)=-\frac{2 s(x, z)}{1+x^{2}+z^{2}}, \quad c(x, z)=k^{2} n(x)^{2}-s \frac{2-s\left(x^{2}+z^{2}\right)}{\left(1+x^{2}+z^{2}\right)^{2}}
$$

Since $\Psi \in W^{2,2}\left(\mathbb{R}^{2}\right)$, by Theorem 8.10 in [41], we have that $\Psi \in W^{3,2}\left(H_{+}\right)$where $H_{+}=\{(x, z) \in$ $\left.\mathbb{R}^{2}: x \geqslant h\right\}$. Again, by the Sobolev imbedding theorem, $|\nabla \Psi|$ is bounded in $H_{+}$and hence the second limit in (21) holds as $x \rightarrow+\infty$. The same limit as $x \rightarrow-\infty$ holds by a similar argument.

\subsection{The radiation condition and uniqueness theorem}

We consider a solution $u$ of (5) and define

$$
u_{l}(x, z)=e\left(x, \gamma_{l}\right) U\left(z, \gamma_{l}\right), \quad l=1, \ldots, M
$$

with $e\left(x, \gamma_{l}\right)$ given by (17) and where

$$
U\left(z, \gamma_{l}\right)=\int_{-\infty}^{\infty} u(\xi, z) e\left(\xi, \gamma_{l}\right) \mathrm{d} \xi, \quad l=1, \ldots, M
$$

The remainder part of $u$ is

$$
u_{0}(x, z)=u(x, z)-\sum_{l=1}^{M} u_{l}(x, z)
$$

We introduce a one-parameter family of sets $\Omega_{R}, R>0$, such that $\partial \Omega_{R}$ are level sets of a convex and coercive function $d(x, z)$, i.e. $\Omega_{R}=\left\{(x, z) \in \mathbb{R}^{2}: d(x, z) \leqslant R\right\}$. 


\section{G. CIRAOLO AND R. MAGNANINI}

With these notations, we state our radiation condition for a solution $u$ of (5):

$$
\sum_{l=0}^{M} \int_{0}^{\infty} \int_{\partial \Omega_{R}}\left|\frac{\partial u_{l}}{\partial v}-i \beta_{l} u_{l}\right|^{2} \mathrm{~d} \ell \mathrm{d} R<+\infty
$$

with $\beta_{0}=k n_{\mathrm{cl}}$ and $\beta_{l}, l=1, \ldots, M$, given by (19).

Note that, when $n \equiv 1$, we can choose $\Omega_{R}=B_{R}$ and (25) reduces to (4), since in such a case the guided components are not present.

The main result of this paper follows.

\section{Theorem 2.3}

There is at most one solution of (5) that satisfies (25) and belongs to $u \in L^{2, s}\left(\mathbb{R}^{2}\right), s<-1$.

\section{Remark 2.4}

As it will be clear, it is not necessary to specify further the sets $\Omega_{R}$ in (25) to get uniqueness of a solution of (5). This means that Theorem 2.3 holds for any choice of one-parameter families of sets $\Omega_{R}$ satisfying the above mentioned assumptions.

Of course, a solution of (5) satisfying (25) may not exist for an arbitrary choice of the sets $\Omega_{R}$. However, a flexibility in the choice of the $\Omega_{R}$ 's could be useful in the development of numerical algorithms. In Section 3, we shall choose a special family of sets $\Omega_{R}$ and prove that, with this choice, the solution of (5) given by (7)-(8) satisfies (25).

We also note that it is not necessary to choose the same sets $\Omega_{R}$ in each addendum in (25); Theorem 2.3 still holds if we replace (25) by the more general radiation condition

$$
\sum_{l=0}^{M} \int_{0}^{\infty} \int_{\partial \Omega_{R}^{(l)}}\left|\frac{\partial u_{l}}{\partial v}-\mathrm{i} \beta_{l} u_{l}\right|^{2} \mathrm{~d} \ell \mathrm{d} R<+\infty
$$

where $\Omega_{R}^{(l)}, l=0,1, \ldots, M$, are one-parameter families satisfying the above mentioned assumptions.

Theorem 2.3 is based on Lemma 2.5 and Theorem 2.6 below.

\section{Lemma 2.5}

Let $\beta \in \mathbb{R}$ and $u$ be a weak solution of (20). Then

$$
\int_{\partial \Omega}\left|\frac{\partial u}{\partial v}-\mathrm{i} \beta u\right|^{2} \mathrm{~d} \ell=\int_{\partial \Omega}\left(\left|\frac{\partial u}{\partial v}\right|^{2}+\beta^{2}|u|^{2}\right) \mathrm{d} \ell
$$

for every $\Omega \subset \mathbb{R}^{2}$ bounded and sufficiently smooth.

\section{Proof}

Since $u$ is a weak solution of (20), by Theorem 8.8 in [41], we obtain the necessary regularity to infer that

$$
\begin{aligned}
\int_{\partial \Omega} \bar{u} \frac{\partial u}{\partial v} \mathrm{~d} \ell & =\int_{\Omega} \operatorname{div}(\bar{u} \nabla u) \mathrm{d} x \mathrm{~d} z=\int_{\Omega}\left\{|\nabla u|^{2}+\bar{u} \Delta u\right\} \mathrm{d} x \mathrm{~d} z \\
& =\int_{\Omega}\left\{|\nabla u|^{2}-k^{2} n(x)^{2}|u|^{2}\right\} \mathrm{d} x \mathrm{~d} z
\end{aligned}
$$


Therefore,

$$
\operatorname{Im} \int_{\partial \Omega} \bar{u} \frac{\partial u}{\partial v} \mathrm{~d} \ell=0
$$

which easily implies (27).

\section{Theorem 2.6}

Let $u \in L^{2, s}\left(\mathbb{R}^{2}\right)$ be a weak solution of (20) satisfying (25). Then

$$
\sum_{l=0}^{M} \int_{0}^{+\infty} \mathrm{d} R \int_{\partial \Omega_{R}}\left[\left|\frac{\partial u_{l}}{\partial v}\right|^{2}+\beta_{l}^{2}\left|u_{l}\right|^{2}\right] \mathrm{d} \ell<+\infty
$$

and, in particular,

$$
\int_{\mathbb{R}^{2}}\left|u_{l}\right|^{2} \mathrm{~d} x \mathrm{~d} z<+\infty
$$

for every $l=0,1, \ldots, M$.

Proof

By Lemma 2.5, it is enough to prove that each $u_{l}, l=0,1, \ldots, M$, satisfies (20). Then, (28) and (29) will follow from (27) and (25).

Suppose $l \geqslant 1$. Since

$$
-\int_{\mathbb{R}^{2}} \nabla u \cdot \nabla \varphi \mathrm{d} x \mathrm{~d} z+k^{2} \int_{\mathbb{R}^{2}} n(x)^{2} u \varphi \mathrm{d} x \mathrm{~d} z=0
$$

for every $\varphi \in H^{1}\left(\mathbb{R}^{2}\right)$, we choose $\varphi(x, z)=e\left(x, \gamma_{l}\right) \eta(z)$ with $\eta \in C_{0}^{1}(\mathbb{R})$ and obtain

$$
\begin{aligned}
& -\int_{\mathbb{R}^{2}}\left[u_{x}(x, z) e^{\prime}\left(x, \gamma_{l}\right) \eta(z)+u_{z}(x, z) e\left(x, \gamma_{l}\right) \eta^{\prime}(z)\right] \mathrm{d} x \mathrm{~d} z \\
& \quad+k^{2} \int_{\mathbb{R}^{2}} n(x)^{2} u(x, z) e\left(x, \gamma_{l}\right) \eta(z) \mathrm{d} x \mathrm{~d} z=0
\end{aligned}
$$

an integration by parts and Lemma 2.2 then give

$$
\begin{aligned}
& \int_{\mathbb{R}^{2}} u(x, z) e^{\prime \prime}\left(x, \gamma_{l}\right) \eta(z) \mathrm{d} x \mathrm{~d} z-\int_{\mathbb{R}^{2}} u_{z}(x, z) e\left(x, \gamma_{l}\right) \eta^{\prime}(z) \mathrm{d} x \mathrm{~d} z \\
& \quad+k^{2} \int_{\mathbb{R}^{2}} n(x)^{2} u(x, z) e\left(x, \gamma_{l}\right) \eta(z) \mathrm{d} x \mathrm{~d} z=0
\end{aligned}
$$

Since $e\left(x, \gamma_{l}\right)$ satisfies (10), we obtain

$$
-\int_{\mathbb{R}^{2}} u_{z}(x, z) e\left(x, \gamma_{l}\right) \eta^{\prime}(z) \mathrm{d} x \mathrm{~d} z+\left(k^{2} n_{*}^{2}-\gamma_{l}\right) \int_{\mathbb{R}^{2}} u(x, z) e\left(x, \gamma_{l}\right) \eta(z) \mathrm{d} x \mathrm{~d} z=0
$$

and thus, from (23),

$$
-\int_{\mathbb{R}} U_{z}\left(z, \gamma_{l}\right) \eta^{\prime}(z) \mathrm{d} z+\left(k^{2} n_{*}^{2}-\gamma_{l}\right) \int_{\mathbb{R}} U\left(z, \gamma_{l}\right) \eta(z) \mathrm{d} z=0
$$

for every $\eta \in C_{0}^{1}(\mathbb{R})$. 


\section{G. CIRAOLO AND R. MAGNANINI}

Together with (10), this formula implies that each $u_{l}(x, z), l=1, \ldots, M$, given by (22), is a weak solution of (20). In fact, for $\varphi(x, z)=\psi(x) \eta(z)$ with $\psi, \eta \in C_{0}^{1}(\mathbb{R})$, integration by parts gives

$$
\begin{aligned}
& -\int_{\mathbb{R}^{2}} \nabla u_{l}(x, z) \cdot \nabla \varphi(x, z) \mathrm{d} x \mathrm{~d} z+k^{2} \int_{\mathbb{R}^{2}} n(x)^{2} u_{l}(x, z) \varphi(x, z) \mathrm{d} x \mathrm{~d} z \\
& =\int_{\mathbb{R}}\left\{e^{\prime \prime}\left(x, \gamma_{l}\right)+\left[\gamma_{l}-q(x)\right] e\left(x, \gamma_{l}\right)\right\} \psi(x) \mathrm{d} x \int_{\mathbb{R}} U\left(z, \gamma_{l}\right) \eta(z) \mathrm{d} z \\
& \quad+\int_{\mathbb{R}} e\left(x, \gamma_{l}\right) \psi(x) \mathrm{d} x \int_{\mathbb{R}}\left[-U_{z}\left(z, \gamma_{l}\right) \eta^{\prime}(z)+\left(k^{2} n_{*}^{2}-\gamma_{l}\right) U\left(z, \gamma_{l}\right)\right] \eta(z) \mathrm{d} z=0
\end{aligned}
$$

the same conclusion holds for any $\varphi \in C_{0}^{1}\left(\mathbb{R}^{2}\right)$, by a density argument.

Since $u$ and $u_{l}, l=1, \ldots, M$, now satisfy (20), the same holds for $u_{0}$. Thus, as already mentioned, we can apply Lemma 2.5 to each $u_{l}, l=0,1, \ldots, M$, and obtain

$$
\sum_{l=0}^{M} \int_{\partial \Omega_{R}}\left|\frac{\partial u_{l}}{\partial v}-\mathrm{i} \beta_{l} u_{l}\right|^{2} \mathrm{~d} \ell=\sum_{l=0}^{M} \int_{\partial \Omega_{R}}\left(\left|\frac{\partial u_{l}}{\partial v}\right|^{2}+\beta_{l}^{2}\left|u_{l}\right|^{2}\right) \mathrm{d} \ell
$$

for every $R>0$, and then since $u$ satisfies (25), we get (28) and (29).

\subsection{Proof of Theorem 2.3}

Let $u_{1}$ and $u_{2}$ be two solutions; $u=u_{1}-u_{2}$ satisfies (20) and (25).

From Theorem 2.6 we have that $u \in L^{2}\left(\mathbb{R}^{2}\right)$ and, by using Lemmas A.1 and A.3 in [39], we get $u \in H^{2}\left(\mathbb{R}^{2}\right)$. Therefore, $u(x, \cdot) \in L^{2}(\mathbb{R})$ for almost every $x \in \mathbb{R}$, and the same holds for $u_{x}(x, \cdot)$ and $u_{x x}(x, \cdot)$. Hence, we can transform (20) by using the Fourier transform in the $z$-coordinate

$$
\hat{u}(x, t)=\int_{-\infty}^{+\infty} u(x, z) \mathrm{e}^{-\mathrm{i} z t} \mathrm{~d} z \quad \text { for a.e. } x \in \mathbb{R}
$$

and obtain

$$
\hat{u}_{x x}(x, t)+\left[k^{2} n(x)^{2}-t^{2}\right] \hat{u}(x, t)=0, \quad \text { a.e. } x \in \mathbb{R}
$$

From Fubini-Tonelli's theorem, the integrals

$$
\int_{\mathbb{R}^{2}}|\hat{u}(x, t)|^{2} \mathrm{~d} x \mathrm{~d} t, \quad \int_{-\infty}^{+\infty} \mathrm{d} t \int_{-\infty}^{+\infty}|\hat{u}(x, t)|^{2} \mathrm{~d} x \quad \text { and } \quad \int_{-\infty}^{+\infty} \mathrm{d} x \int_{-\infty}^{+\infty}|\hat{u}(x, t)|^{2} \mathrm{~d} t
$$

have the same value, finite or infinite.

Since $u(x, \cdot)$ belongs to $L^{2}(\mathbb{R})$ for almost every $x \in \mathbb{R}$, the same holds for $\hat{u}(x, \cdot)$ and, furthermore, we have

$$
\int_{-\infty}^{+\infty}|\hat{u}(x, t)|^{2} \mathrm{~d} t=2 \pi \int_{-\infty}^{+\infty}|u(x, z)|^{2} \mathrm{~d} z \quad \text { a.e. } x \in \mathbb{R}
$$


By integrating the above equation and using Fubini-Tonelli's theorem, we obtain

$$
\begin{aligned}
\int_{\mathbb{R}^{2}}|\hat{u}(x, t)|^{2} \mathrm{~d} x \mathrm{~d} t & =\int_{-\infty}^{+\infty} \mathrm{d} x \int_{-\infty}^{+\infty}|\hat{u}(x, t)|^{2} \mathrm{~d} t=2 \pi \int_{-\infty}^{+\infty} \mathrm{d} x \int_{-\infty}^{+\infty}|u(x, z)|^{2} \mathrm{~d} z \\
& =2 \pi \int_{\mathbb{R}^{2}}|u(x, z)|^{2} \mathrm{~d} x \mathrm{~d} z<+\infty
\end{aligned}
$$

Therefore, $\hat{u}(\cdot, t) \in L^{2}(\mathbb{R})$ for almost every $t \in \mathbb{R}$.

From (31), it follows that

$$
\hat{u}(x, t)=a(t) \cos \sqrt{\lambda-d^{2}}(x-h)+b(t) \sin \sqrt{\lambda-d^{2}}(x-h) \quad \text { for } x>h
$$

where $\lambda=k^{2} n_{*}^{2}-t^{2}$ and $d^{2}=k^{2}\left(n_{*}^{2}-n_{\mathrm{cl}}^{2}\right)$. Since

$$
\int_{-\infty}^{+\infty}|\hat{u}(x, t)|^{2} \mathrm{~d} x \geqslant \int_{h}^{+\infty}|\hat{u}(x, t)|^{2} \mathrm{~d} x
$$

we obtain that $\hat{u}(x, t)$ cannot be identically zero only for some values $t=\lambda_{m}^{j} \in\left(0, d^{2}\right]$ and, furthermore, in that case

$$
\hat{u}(x, t)=a(t) v_{s}\left(x, \lambda_{s}^{m}\right)+b(t) v_{a}\left(x, \lambda_{a}^{m}\right)
$$

Hence, for some $A, B \in \mathbb{R}$ we should have

$$
u(x, z)=A Z_{s}(z) v_{s}\left(x, \lambda_{s}^{m}\right)+B Z_{a}(z) v_{a}\left(x, \lambda_{a}^{m}\right)
$$

where $Z_{j}(z)=\mathrm{e}^{ \pm z \sqrt{k^{2} n_{*}^{2}-\lambda_{m}^{j}}}$, because $u$ is a solution of (20). Since $u(x, \cdot) \in L^{2}(\mathbb{R})$, then both $A$ and $B$ must be zero and hence $u \equiv 0$ on $\mathbb{R}^{2}$.

\section{THE SPECTRUM-BASED SOLUTION SATISFIES THE RADIATION CONDITION}

Throughout this section we will assume that $n$ is a function of bounded variation.

It will be useful to introduce the following function:

$$
[x]_{h}= \begin{cases}x+h, & x<-h \\ 0, & -h \leqslant x \leqslant h \\ x-h, & x>h\end{cases}
$$

This section is devoted to the proof of the following result.

\section{Theorem 3.1}

Let $f \in L^{2}\left(\mathbb{R}^{2}\right)$ be such that $f \equiv 0$ a.e. outside a compact subset of $\mathbb{R}^{2}$. Then, the spectrum-based solution (7) of (5) is the only solution in $L^{2, s}\left(\mathbb{R}^{2}\right), s<-1$, such that

$$
\int_{0}^{\infty} \int_{\partial \Omega_{R}}\left|\frac{\partial u_{0}}{\partial v}-\mathrm{i} \beta_{0} u_{0}\right|^{2} \mathrm{~d} \ell \mathrm{d} R+\sum_{l=1}^{M} \int_{0}^{\infty} \int_{\partial Q_{R}}\left|\frac{\partial u_{l}}{\partial v}-\mathrm{i} \beta_{l} u_{l}\right|^{2} \mathrm{~d} \ell \mathrm{d} R<+\infty
$$




\section{G. CIRAOLO AND R. MAGNANINI}

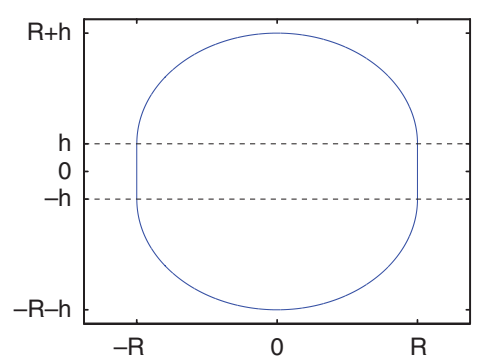

Figure 1. The set $\Omega_{R}$.

where $\Omega_{R}$ is given by

$$
\Omega_{R}=\left\{(x, z) \in \mathbb{R}^{2}:[x]_{h}^{2}+z^{2} \leqslant R^{2}\right\}
$$

(see Figure 1) and $Q_{R}=\left\{(x, z) \in \mathbb{R}^{2}:|x|,|z| \leqslant R\right\}$.

Remark 3.2

At the cost of extra computations, it may be proved that Theorem 3.1 also holds if we replace (26) by the more compact condition (25) with $\Omega_{R}$ given by (34).

We shall break the proof of Theorem 3.1 up into three steps. First, in Section 3.1, we will derive a handier representation of the radiating part $G^{\text {rad }}$ of Green's function, as a suitable contour integral (see Lemma 3.3). Then, in Section 3.2, we will prove a uniform asymptotic expansion for the quantity $\partial G^{\mathrm{rad}} / \partial v-\mathrm{i} \beta_{0} G^{\mathrm{rad}}$ on the sets $\partial \Omega_{R}$. Such an expansion will be used in Section 3.3 to carry out the proof of Theorem 3.1, where we also test the radiation condition on the guided components of $u$.

\subsection{Representing $G^{\mathrm{rad}}$ as a contour integral}

We introduce the following functions:

$$
\{x\}_{h}=x-[x]_{h}
$$

with $[x]_{h}$ given by (32), and, for $\tau \in \mathbb{C}$,

$$
\Phi_{j}(x, \tau)=\phi_{j}\left(\{x\}_{h}, d^{2}+\tau^{2}\right)+\frac{\phi_{j}^{\prime}\left(\{x\}_{h}, d^{2}+\tau^{2}\right)}{\mathrm{i} \tau}, \quad j \in\{s, a\}
$$

With these notations, (11) and (14) take the more compact forms:

$$
v_{j}\left(x, d^{2}+\tau^{2}\right)=\frac{1}{2}\left\{\Phi_{j}(x, \tau) \mathrm{e}^{\mathrm{i} \tau[x]_{h}}+\Phi_{j}(x,-\tau) \mathrm{e}^{-\mathrm{i} \tau[x]_{h}}\right\}
$$

and

$$
\sigma_{j}\left(d^{2}+\tau^{2}\right)=\frac{1}{\Phi_{j}(h, \tau) \Phi_{j}(h,-\tau)}
$$

for $j \in\{s, a\}$. 


\section{UNIQUENESS FOR 2-D WAVEGUIDES}

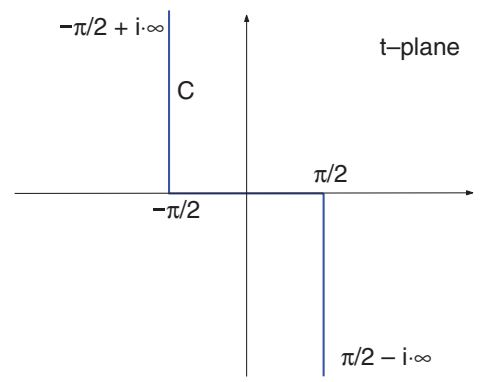

Figure 2. The contour $\mathscr{C}$.

\section{Lemma 3.3}

Let $\mathscr{C}$ be the contour from $-\frac{\pi}{2}+\mathrm{i} \cdot \infty$ to $\frac{\pi}{2}-\mathrm{i} \cdot \infty$ shown in Figure 2 and let $G^{\text {rad }}$ be the function in (13). Then,

$$
G^{\mathrm{rad}}=\sum_{j \in\{s, a\}} \int_{\mathscr{C}}\left[A_{j}^{+}(x, \xi ; t) \mathrm{e}^{\mathrm{i} \beta_{0} \alpha_{+}(x, z ; \xi, \zeta ; t)}+A_{j}^{-}(x, \xi ; t) \mathrm{e}^{\mathrm{i} \beta_{0} \alpha_{-}(x, z ; \xi, \zeta ; t)}\right] \mathrm{d} t
$$

with

$$
A_{j}^{ \pm}(x, \xi ; t)=\frac{1}{8 \pi i} \frac{\Phi_{j}\left(x, \beta_{0} \sin t\right) \Phi_{j}\left(\xi, \pm \beta_{0} \sin t\right)}{\Phi_{j}\left(h, \beta_{0} \sin t\right) \Phi_{j}\left(h,-\beta_{0} \sin t\right)}
$$

and

$$
\alpha_{ \pm}(x, z ; \xi, \zeta ; t)=\left([x]_{h} \pm[\xi]_{h}\right) \sin t+|z-\zeta| \cos t
$$

$t \in \mathbb{C}$, and where $\Phi_{j}, j \in\{s, a\}$, is given by (35). In particular, the following equivalent expression for $G^{\text {rad }}$ will also be useful:

$$
G^{\mathrm{rad}}=\int_{\mathscr{C}} g(x, \xi ; t) \mathrm{e}^{\mathrm{i} \beta_{0}\left([x]_{h} \sin t+|z-\zeta| \cos t\right)} \mathrm{d} t
$$

where

$$
g(x, \xi ; t)=\sum_{j \in\{s, a\}}\left[A_{j}^{+}(x, \xi ; t) \mathrm{e}^{\mathrm{i}[\xi] h \sin t}+A_{j}^{-}(x, \xi ; t) \mathrm{e}^{-\mathrm{i}[\xi] / \sin t}\right]
$$

(Note that $g$ does not depend on $x$ for $|x| \geqslant h$.)

Proof

We first take (13) and make the change of variable $\tau=\sqrt{\lambda-d^{2}}$ to obtain

$$
G^{\mathrm{rad}}=\frac{1}{4 \pi \mathrm{i}} \sum_{j \in\{s, a\}} \int_{-\infty}^{+\infty} \frac{\mathrm{e}^{\mathrm{i}|z-\zeta| \sqrt{\beta_{0}^{2}-\tau^{2}}}}{\sqrt{\beta_{0}^{2}-\tau^{2}}} v_{j}\left(x, \tau^{2}+d^{2}\right) v_{j}\left(\xi, \tau^{2}+d^{2}\right) \sigma_{j}\left(\tau^{2}+d^{2}\right) \mathrm{d} \tau
$$




\section{G. CIRAOLO AND R. MAGNANINI}

here, we also used the fact that all the relevant quantities subject to integration are even functions of $\tau$. With the help of (36) and (37), and simple manipulations, we can infer that

$$
\begin{aligned}
G^{\mathrm{rad}}= & \frac{1}{8 \pi \mathrm{i}} \sum_{j \in\{s, a\}} \int_{-\infty}^{+\infty}\left\{\frac{\Phi_{j}(x, \tau) \Phi_{j}(\xi, \tau)}{\Phi_{j}(h, \tau) \Phi_{j}(h,-\tau)} \mathrm{e}^{\mathrm{i}}\left[\tau\left([x]_{h}+[\xi] h\right)+|z-\zeta| \sqrt{\beta_{0}^{2}-\tau^{2}}\right]\right. \\
& \left.+\frac{\Phi_{j}(x, \tau) \Phi_{j}(\xi,-\tau)}{\Phi_{j}(h, \tau) \Phi_{j}(h,-\tau)} \mathrm{e}^{\mathrm{i}\left[\tau\left([x]_{h}-[\xi]_{h}\right)+|z-\zeta| \sqrt{\left.\beta_{0}^{2}-\tau^{2}\right]}\right.}\right\} \mathrm{d} \tau
\end{aligned}
$$

The conclusion is then readily obtained by splitting up the interval of integration into the three intervals $\left(-\infty,-\beta_{0}\right),\left[-\beta_{0}, \beta_{0}\right]$ and $\left(\beta_{0},+\infty\right)$ and by subsequently making the change of variable $\tau=\beta_{0} \sin t$, with $t \in \mathscr{C}$.

\section{Lemma 3.4}

For every $\xi, \zeta$ fixed, we have

$$
\frac{\partial G^{\mathrm{rad}}}{\partial x}=\mathrm{i} \beta_{0} \int_{\mathscr{C}} g(h \operatorname{sign} x, \xi ; t) \sin t \mathrm{e}^{\mathrm{i} \beta_{0}([x] h \sin t+|z-\zeta| \cos t)} \mathrm{d} t
$$

for $|x| \geqslant h$ and $z \neq \zeta$

$$
\frac{\partial G^{\mathrm{rad}}}{\partial z}=\mathrm{i} \beta_{0} \operatorname{sign}(z-\zeta) \int_{\mathscr{C}} g(x, \xi ; t) \cos t \mathrm{e}^{\mathrm{i} \beta_{0}\left([x]_{h} \sin t+|z-\zeta| \cos t\right)} \mathrm{d} t
$$

for $z \neq \zeta$.

In particular, on the set $(0, \zeta)+\partial \Omega_{R}$ given by (34), we have

$$
\frac{\partial G^{\mathrm{rad}}}{\partial v}-\mathrm{i} \beta_{0} G^{\mathrm{rad}}=\mathrm{i} \beta_{0} \int_{\mathscr{C}} g(x, \xi ; t)[\cos t-1] \mathrm{e}^{\mathrm{i} \beta_{0} R \cos t} \mathrm{~d} t
$$

for $z-\zeta=R$ and $|x| \leqslant h$, and

$$
\frac{\partial G^{\mathrm{rad}}}{\partial v}-\mathrm{i} \beta_{0} G^{\mathrm{rad}}=\mathrm{i} \beta_{0} \int_{\mathscr{C}} g(h, \xi ; t)[\cos (t-\vartheta)-1] \mathrm{e}^{\mathrm{i} \beta_{0} R \cos (t-\vartheta)} \mathrm{d} t
$$

where $v$ is the normal to $(0, \zeta)+\partial \Omega_{R}$ and we have set $[x]_{h}=R \sin \vartheta$ and $z-\zeta=R \cos \vartheta$ with $\vartheta \in[0, \pi / 2)$ and $R>h$.

Formulas analogous to (41) hold for the remaining values of $\vartheta$ in $[-\pi, \pi)$.

\section{Proof}

Since $z \neq \zeta$ and $\operatorname{Im}\left([x]_{h} \sin t+|z-\zeta| \cos t\right) \rightarrow+\infty$ as $t \rightarrow \infty$ on $\mathscr{C}$, the integrands in (40a) and (40b) vanish exponentially as $t \rightarrow \infty$ on $\mathscr{C}$, since $g$ is bounded (see Lemma A.3). Thus, (40a) and (40b) follow from an application of Lebesgue's dominated convergence theorem.

\subsection{Uniform asymptotic estimates for $\partial G^{\mathrm{rad}} / \partial v-\mathrm{i} \beta_{0} G^{\mathrm{rad}}$}

Aiming to estimate, as $R \rightarrow \infty$, the function $\partial G^{\mathrm{rad}} / \partial v-\mathrm{i} \beta_{0} G^{\mathrm{rad}}$ given by (41), we need to deform the contour $\mathscr{C}$ to a more convenient one. 


\section{UNIQUENESS FOR 2-D WAVEGUIDES}

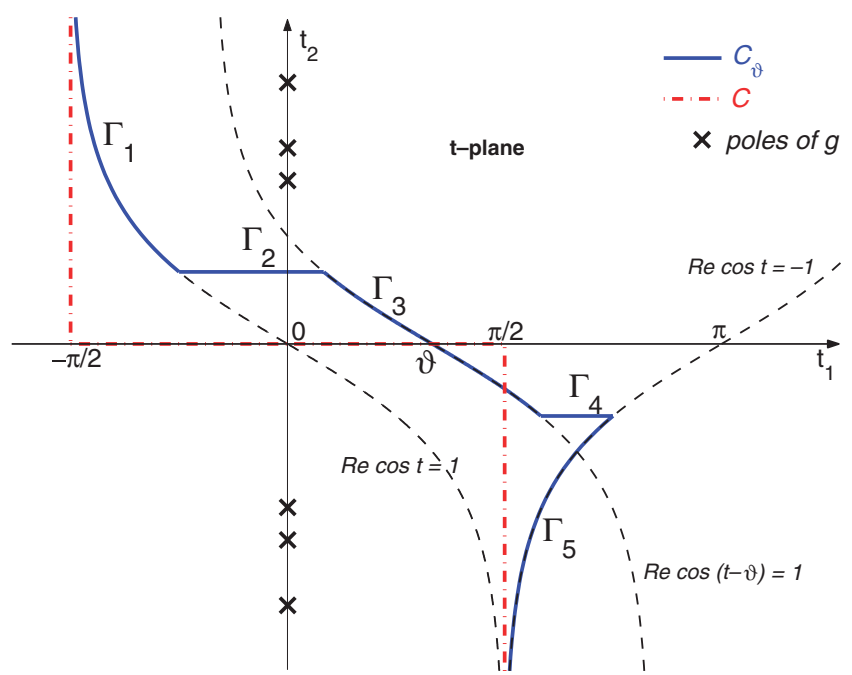

Figure 3. The contour $\mathscr{C}$.

Without loss of generality we can assume that $\vartheta \in[0, \pi / 2]$. We define the new contour $\mathscr{C}_{\vartheta}$ (see Figure 3) as follows:

$$
\mathscr{C}_{\vartheta}=\bigcup_{j=1}^{5} \Gamma_{j}
$$

where

$$
\delta_{1}=\arccos \frac{2 \beta_{0}}{\sqrt{4 \beta_{0}^{2}+d^{2}-\gamma_{M}}}, \quad \delta_{2}=\operatorname{arcsinh} \frac{\sqrt{d^{2}-\gamma_{M}}}{2 \beta_{0}}
$$

(note that $\cos \delta_{1} \cosh \delta_{2}=1$ ) and

$$
\begin{aligned}
& \Gamma_{1}=\left\{t=t_{1}+\mathrm{i} t_{2} \in \mathbb{C}: \operatorname{Re}(\cos t)=1, \operatorname{Im}(\cos t) \geqslant 0,-\frac{\pi}{2}<t_{1} \leqslant-\delta_{1}, t_{2} \geqslant \delta_{2}\right\} \\
& \Gamma_{2}=\left\{t \in \mathbb{C}:-\delta_{1} \leqslant t_{1} \leqslant-\delta_{1}+\vartheta, t_{2}=\delta_{2}\right\} \\
& \Gamma_{3}=\left\{t \in \mathbb{C}: \operatorname{Re}[\cos (t-\vartheta)]=1, \operatorname{Im}[\cos (t-\vartheta)] \geqslant 0,\left|t_{1}-\vartheta\right| \leqslant \delta_{1},\left|t_{2}\right| \leqslant \delta_{2}\right\} \\
& \Gamma_{4}=\left\{t \in \mathbb{C}: \delta_{1}+\vartheta \leqslant t_{1} \leqslant \pi-\delta_{1}, t_{2}=-\delta_{2}\right\} \\
& \Gamma_{5}=\left\{t \in \mathbb{C}: \operatorname{Re}(\cos t)=-1, \operatorname{Im}(\cos t) \geqslant 0, \frac{\pi}{2}<t_{1} \leqslant \pi-\delta_{1}, t_{2} \leqslant-\delta_{2}\right\}
\end{aligned}
$$

This choice of $\mathscr{C}_{\vartheta}$ is suggested by the following three remarks:

(i) $\mathscr{C} \cup \mathscr{C}_{\vartheta}$ does not contain in its interior the poles of $g$ (which correspond to the guided part (12) of $G$ ).

(ii) $\Gamma_{3}$ is part of the steepest descent path of $\cos (t-\vartheta)$.

(iii) $\Gamma_{1}, \Gamma_{2}, \Gamma_{4}, \Gamma_{5}$ are chosen to complete the contour $\mathscr{C} \cup \mathscr{C}_{\vartheta}$ and to fulfill Lemma 3.5 below. 


\section{G. CIRAOLO AND R. MAGNANINI}

By (i), it is clear that we can express

$$
\frac{\partial G^{\mathrm{rad}}}{\partial v}-\mathrm{i} \beta_{0} G^{\mathrm{rad}}=\mathrm{i} \beta_{0} \int_{\mathscr{C}_{0}} g(x, \xi ; t)[\cos t-1] \mathrm{e}^{\mathrm{i} \beta_{0} R \cos t} \mathrm{~d} t
$$

for $|x| \leqslant h$, and

$$
\frac{\partial G^{\mathrm{rad}}}{\partial v}-\mathrm{i} \beta_{0} G^{\mathrm{rad}}=\mathrm{i} \beta_{0} \int_{\mathscr{C}_{\vartheta}} g(h, \xi ; t)[\cos (t-\vartheta)-1] \mathrm{e}^{\mathrm{i} \beta_{0} R \cos (t-\vartheta)} \mathrm{d} t
$$

for $x \geqslant h$.

Lemma 3.5

Let $(x, z) \in(0, \zeta)+\partial \Omega_{R}$. The following estimates hold for $R \rightarrow \infty$ :

$$
\frac{\partial G^{\mathrm{rad}}}{\partial v}-\mathrm{i} \beta_{0} G^{\mathrm{rad}}=\mathrm{i} \beta_{0} \int_{\Gamma_{3}} g(x, \xi ; t)[\cos t-1] \mathrm{e}^{\mathrm{i} \beta_{0} R \cos t} \mathrm{~d} t+\mathcal{O}\left(\mathrm{e}^{-c \beta_{0} R}\right)
$$

for $|x| \leqslant h$, and

$$
\frac{\partial G^{\mathrm{rad}}}{\partial v}-\mathrm{i} \beta_{0} G^{\mathrm{rad}}=\mathrm{i} \beta_{0} \int_{\Gamma_{3}} g(h, \xi ; t)[\cos (t-\vartheta)-1] \mathrm{e}^{\mathrm{i} \beta_{0} R \cos (t-\vartheta)} \mathrm{d} t+\mathcal{O}\left(\mathrm{e}^{-c \beta_{0} R}\right)
$$

for $x \geqslant h, \vartheta \in[0, \pi / 2]$, where

$$
c=\sqrt{\frac{d^{2}-\gamma_{M}}{4 \beta_{0}^{2}+d^{2}-\gamma_{M}}} \min \left(1, \frac{\sqrt{d^{2}-\gamma_{M}}}{2 \beta_{0}}\right)
$$

Proof

We shall prove only (42b) since (42a) follows analogously. We preliminarily observe that

$$
\operatorname{Im} \cos (t-\vartheta) \geqslant c
$$

for $t \in \Gamma_{1}, \Gamma_{2}, \Gamma_{4}, \Gamma_{5}$ and $\vartheta \in[0, \pi / 2]$. From (43), we easily obtain that

$$
\left|\int_{\Gamma_{j}} g(h, \xi ; t)[\cos (t-\vartheta)-1] \mathrm{e}^{\mathrm{i} \beta_{0} R \cos (t-\vartheta)} \mathrm{d} t\right| \leqslant \frac{K \pi}{2}\left(\cosh \delta_{2}+1\right) \mathrm{e}^{-c \beta_{0} R}, \quad j=2,4
$$

where $K$ is a bound for $g$ (see Lemma A.3). Thus, it remains to prove that

$$
\int_{\Gamma_{j}} g(h, \xi ; t)[\cos (t-\vartheta)-1] \mathrm{e}^{\mathrm{i} \beta_{0} R \cos (t-\vartheta)} \mathrm{d} t=\mathcal{O}\left(\mathrm{e}^{-c \beta_{0} R}\right), \quad j=1,5
$$

uniformly as $R \rightarrow \infty$, for $\vartheta \in[0, \pi / 2]$. We carry out the details for $j=1$, the case $j=5$ is completely analogous. We first use Lemma A.2 to express that

$$
\int_{\Gamma_{1}} g(h, \xi ; t)[\cos (t-\vartheta)-1] \mathrm{e}^{\mathrm{i} \beta_{0} R \cos (t-\vartheta)} \mathrm{d} t=J(R)+\mathcal{O}\left(\mathrm{e}^{-c \beta_{0} R}\right)
$$


since (43) holds; here,

$$
J(R)=\mathrm{i} \beta_{0} \int_{\Gamma_{1}}\left(1+\frac{\mathrm{i}}{2 \beta_{0} \sin t} \int_{\{\xi\}_{h}}^{h} p(y) \mathrm{d} y\right)[\cos (t-\vartheta)-1] \mathrm{e}^{\mathrm{i} \beta_{0}[R \cos (t-\vartheta)+(h-\xi) \sin t]} \mathrm{d} t
$$

with $p(y)=d^{2}-q(y)$. Let $\psi(t)=R \cos (t-\vartheta)+(h-\xi) \sin t$ and $\delta=\delta_{1}+\mathrm{i} \delta_{2}$; an integration by parts yields

$$
\begin{aligned}
J(R)= & \frac{\mathrm{e}^{\mathrm{i} \beta_{0} \psi(\delta)}}{\psi^{\prime}(\delta)}\left(1+\frac{\mathrm{i}}{2 \beta_{0} \sin \delta} \int_{\{\xi\}_{h}}^{h} p(y) \mathrm{d} y\right)[1-\cos (\delta-\vartheta)] \\
& +\int_{\Gamma_{1}} \frac{\mathrm{e}^{\mathrm{i} \beta_{0} \psi(t)}}{\psi^{\prime}(t)^{2}}\left\{[\cos (t-\vartheta)-1]\left[R-\frac{\mathrm{i} \psi(t)}{2 \beta_{0} \sin t} \int_{\{\xi\}_{h}}^{h} p(y) \mathrm{d} y\right]\right. \\
& \left.+(h-\xi)(\sin t-\sin \vartheta)-\frac{\mathrm{i} \psi^{\prime}(t)(\cos t-\cos \vartheta)}{2 \beta_{0} \sin ^{2} t} \int_{\{\xi\}_{h}}^{h} p(y) \mathrm{d} y\right\} \mathrm{d} t
\end{aligned}
$$

From (43) and since

$$
\begin{aligned}
& \sinh t_{2} \leqslant|\cos t|, \quad|\sin t| \leqslant \cosh t_{2} \\
& |\psi(t)| \leqslant \beta_{0}(R+|h-\xi|) \cosh t_{2}, \quad\left|\psi^{\prime}(t)\right| \geqslant \frac{1}{2} \beta_{0} R \sinh t_{2}
\end{aligned}
$$

for $R \geqslant 2|h-\xi| \operatorname{coth} \delta_{2}$ and $t \in \Gamma_{1}$, we obtain that $J(R)=\mathcal{O}\left(\mathrm{e}^{-c \beta_{0} R}\right)$, as $R \rightarrow \infty$.

Theorem 3.6

On $\partial \Omega_{R}$, we have

$$
\frac{\partial G^{\mathrm{rad}}}{\partial v}-\mathrm{i} \beta_{0} G^{\mathrm{rad}}=\mathcal{O}\left(R^{-3 / 2}\right)
$$

uniformly as $R \rightarrow \infty$.

Proof

First, we estimate the left-hand side of (44) on the sets $(0, \zeta)+\partial \Omega_{R}$. By Lemma 3.5, we need only to estimate the first addendum in (42). We prove the estimate for (42b); the estimate for (42a) follows exactly in the same manner.

Since $\Gamma_{3}$ is part of the steepest descent path, the steepest descent method (see [42]) suggests to change the variables in the first addendum in (42b): by setting $\cos (t-\vartheta)=1+\mathrm{i} y^{2}$, we obtain

$$
\int_{\Gamma_{3}} g(h, \xi ; t)[\cos (t-\vartheta)-1] \mathrm{e}^{\mathrm{i} \beta_{0} R \cos (t-\vartheta)} \mathrm{d} t=-4 \mathrm{ie}^{\mathrm{i} \beta_{0} R} \int_{0}^{y_{0}} y^{2} \mathrm{e}^{-\beta_{0} R y^{2}} \frac{g(h, \xi ; t(y))}{\sqrt{y^{2}-2 i}} \mathrm{~d} y
$$

with $y_{0}=\left(\sin \delta_{1} \sinh \delta_{2}\right)^{1 / 2}$. Thanks to Lemma A.3,

$$
\begin{aligned}
& \left|\int_{\Gamma_{3}} g(h, \xi ; t)[\cos (t-\vartheta)-1] \mathrm{e}^{\mathrm{i} \beta_{0} R \cos (t-\vartheta)} \mathrm{d} t\right| \\
& \quad \leqslant 2 \sqrt{2} K \int_{0}^{y_{0}} y^{2} \mathrm{e}^{-\beta_{0} R y^{2}} \mathrm{~d} y \leqslant K \sqrt{\frac{\pi}{2 \beta_{0}^{3}}} R^{-3 / 2}
\end{aligned}
$$




\section{G. CIRAOLO AND R. MAGNANINI}

where $K$ is a bound of $g$. Therefore, (42b) implies that

$$
\left|\frac{\partial G^{\mathrm{rad}}}{\partial v}-\mathrm{i} \beta_{0} G^{\mathrm{rad}}\right| \leqslant K \sqrt{\frac{\pi}{2 \beta_{0}}} R^{-3 / 2}
$$

on the sets $(0, \zeta)+\partial \Omega_{R}$.

By using exactly the same argument as before, we can prove that the derivatives of $G^{\mathrm{rad}}$ are $\mathcal{O}\left(R^{-1 / 2}\right)$ on the sets $(0, \zeta)+\partial \Omega_{R}$, uniformly as $R \rightarrow \infty$; we reach the conclusion (44) by observing that $v_{\partial \Omega_{R}}-v_{(0, \zeta)+\partial \Omega_{R}}=\mathcal{O}\left(R^{-1}\right)$, as $R \rightarrow \infty$.

\subsection{Proof of Theorem 3.1}

Since $f \in L^{2}\left(\mathbb{R}^{2}\right)$ and $f$ has compact support, from Corollary 5.1 in [39] we have that $u \in L^{2, s}\left(\mathbb{R}^{2}\right)$, $s<-1$. facts:

Thus, it remains to prove that (7) satisfies (25). In order to do it, we shall check the following

(i) if $u$ is given by (7) and $u_{l}, l=1, \ldots, M$, is computed via (22), the remainder part $u_{0}$ of $u$, given by (24), equals the function $u^{\mathrm{rad}}$ in (15).

(ii) $u$ satisfies (33).

We preliminarily note that

$$
\int_{0}^{+\infty} \int_{\partial \Omega_{R}}\left|\frac{\partial u_{0}}{\partial v}-\mathrm{i} \beta_{0} u_{0}\right|^{2} \mathrm{~d} \ell \mathrm{d} R<+\infty
$$

is easily verified thanks to Theorem 3.6.

The following property of orthogonality is useful to check (i).

\section{Lemma 3.7}

Let $e\left(x, \gamma_{l}\right), l=1, \ldots, M$, and $v_{j}(x, \lambda), j \in\{s, a\}$, be the solutions of (10) given by (17) and (11), respectively, with $\lambda>0$. If $\lambda \neq \gamma_{l}$, then

$$
\int_{-\infty}^{+\infty} e\left(x, \gamma_{l}\right) v_{j}(x, \lambda) \mathrm{d} x=0
$$

Proof

We multiply the following equations:

$$
\begin{array}{r}
e^{\prime \prime}\left(x, \gamma_{l}\right)+\left[\gamma_{l}-q(x)\right] e\left(x, \gamma_{l}\right)=0 \\
v^{\prime \prime}(x, \lambda)+[\lambda-q(x)] v(x, \lambda)=0
\end{array}
$$

by $v(x, \lambda)$ and $e\left(x, \gamma_{l}\right)$, respectively, and integrate in $x$ over an interval $(a, b)$. An integration by parts gives

$$
\begin{aligned}
\left(\gamma_{l}-\lambda\right) \int_{a}^{b} e\left(x, \gamma_{l}\right) v(x, \lambda) \mathrm{d} x & =\int_{a}^{b}\left[e\left(x, \gamma_{l}\right) v^{\prime \prime}(x, \lambda)-e^{\prime \prime}\left(x, \gamma_{l}\right) v(x, \lambda)\right] \mathrm{d} x \\
& =\left[e\left(x, \gamma_{l}\right) v^{\prime}(x, \lambda)-e^{\prime}\left(x, \gamma_{l}\right) v(x, \lambda)\right]_{a}^{b}
\end{aligned}
$$


The conclusion follows by observing that $e\left(x, \gamma_{l}\right)$ and its first derivative vanish exponentially as $|x| \rightarrow \infty$, while $v(x, \lambda)$ and $v^{\prime}(x, \lambda)$ are bounded.

Now, by (7), (22) and Lemma 3.7, we have that

$$
u_{l}(x, z)=\int_{\mathbb{R}^{2}} G_{l}^{g}(x, z ; \xi, \zeta) f(\xi, \zeta) \mathrm{d} \xi \mathrm{d} \zeta, \quad l=1, \ldots, M
$$

with $G_{l}^{g}$ given by (18) and thus $u_{0}=u^{\mathrm{rad}}$.

To complete the proof it remains to check (ii) for $l=1, \ldots, M$. When $z$ is large enough, we have

$$
\frac{\partial u_{l}}{\partial v}-\mathrm{i} \beta_{l} u_{l}=0, \quad l=1, \ldots, M
$$

on $\partial Q_{R} \cap\{(x, z):|z|=R\}$, since $\partial / \partial v= \pm \partial / \partial z$. Thanks to (16), we easily find that

$$
\left|\frac{\partial u_{l}}{\partial v}-\mathrm{i} \beta_{l} u_{l}\right|=\mathcal{O}\left(\mathrm{e}^{-R \sqrt{d^{2}-\gamma_{l}}}\right)
$$

as $R \rightarrow \infty$ on $\partial Q_{R} \cap\{(x, z):|x|=R\}$ and thus we obtain that

$$
\int_{0}^{+\infty} \int_{\partial Q_{R}}\left|\frac{\partial u_{l}}{\partial v}-\mathrm{i} \beta_{l} u_{l}\right|^{2} \mathrm{~d} \ell \mathrm{d} R<+\infty, \quad l=1, \ldots, M
$$

which completes the proof.

\section{APPENDIX A: ASYMPTOTIC LEMMAS}

In Lemmas A.1-A.3 we will always assume that $q$ is a function of bounded variation. We note that, under this assumption, the solutions (11) of (10) have absolutely continuous first derivative (see [43]).

\section{Lemma A.1}

Let $T$ be a non-negative number and

$$
p(x)=d^{2}-q(x), \quad x \in \mathbb{R}
$$

Then, the following asymptotic estimates for the functions $\Phi_{s}$ and $\Phi_{a}$ given by (35) hold uniformly as $|\tau| \rightarrow+\infty$, for $x \in \mathbb{R}$ and $|\operatorname{Im} \tau| \leqslant T$ :

$$
\begin{aligned}
& \Phi_{s}(x, \tau)=\left[1+\frac{\mathrm{i}}{2 \tau} \int_{0}^{\{x\}_{h}} p(y) \mathrm{d} y\right] \mathrm{e}^{\mathrm{i} \tau\{x\}_{h}}+\mathcal{O}\left(\frac{1}{|\tau|^{2}}\right) \\
& \Phi_{a}(x, \tau)=\frac{\sqrt{\tau^{2}+d^{2}}}{\mathrm{i} \tau}\left[1+\frac{\mathrm{i}}{2 \tau} \int_{0}^{\{x\}_{h}} p(y) \mathrm{d} y\right] \mathrm{e}^{\mathrm{i} \tau\{x\}_{h}}+\mathcal{O}\left(\frac{1}{|\tau|^{2}}\right)
\end{aligned}
$$




\section{G. CIRAOLO AND R. MAGNANINI}

Proof

(i) First, we prove an estimate for $\phi_{j}, j \in\{s, a\}$. From (10), we know that $\phi_{j}$ satisfies

$$
\phi_{j}^{\prime \prime}(y, \lambda)+\left[\tau^{2}+p(y)\right] \phi_{j}(y, \lambda)=0, \quad y \in[-h, h]
$$

We multiply the above equation by $\sin \tau(x-y)$, integrate by parts twice and obtain the following integral equation:

$$
\phi_{j}(x, \lambda)=\frac{\phi_{j}^{\prime}(0, \lambda)}{\tau} \sin \tau x+\phi_{j}(0, \lambda) \cos \tau x-\frac{1}{\tau} \int_{0}^{x} p(y) \sin (\tau(x-y)) \phi_{j}(y, \lambda) \mathrm{d} y
$$

We set $\eta_{j}(x, \lambda)=\sup _{s \in[0, x]}\left|\phi_{j}(s, \lambda)\right|$. Since $|\sin \tau x|,|\cos \tau x| \leqslant \cosh \tau_{2} x\left(\tau_{2}=\operatorname{Im} \tau\right)$, from the above equation we have that

$$
\begin{aligned}
\eta_{j}(x, \lambda) \leqslant & {\left[\frac{\left|\phi_{j}^{\prime}(0, \lambda)\right|}{|\tau|}+\left|\phi_{j}(0, \lambda)\right|\right] \cosh \tau_{2} x } \\
& +\frac{1}{|\tau|} \int_{0}^{x} p(y) \cosh \tau_{2}(x-y) \eta_{j}(y, \lambda) \mathrm{d} y
\end{aligned}
$$

and, by Gronwall's Lemma (see [44]), we get

$$
\begin{aligned}
\eta_{j}(x, \lambda) \leqslant & {\left[\frac{\left|\phi_{j}^{\prime}(0, \lambda)\right|}{|\tau|}+\left|\phi_{j}(0, \lambda)\right|\right] \mathrm{e}^{1 /|\tau| \int_{0}^{x} p(y) \cosh \tau_{2}(x-y) \mathrm{d} y} } \\
& \times\left\{1+\tau_{2} \int_{0}^{x} \mathrm{e}^{-\int_{0}^{s} p(y) \cosh \tau_{2}(x-y) \mathrm{d} y} \sinh \tau_{2} s \mathrm{~d} s\right\}
\end{aligned}
$$

Since $0 \leqslant p(y) \leqslant d^{2}$, we have that

$$
\eta_{j}(x, \lambda) \leqslant\left[\frac{\left|\phi_{j}^{\prime}(0, \lambda)\right|}{|\tau|}+\left|\phi_{j}(0, \lambda)\right|\right] \cosh \tau_{2} x \exp \left\{\frac{d^{2} \sinh \tau_{2} x}{|\tau| \tau_{2}}\right\}
$$

If we assume $|\tau| \geqslant d$ and $x \in[-h, h]$, we finally get

$$
\left|\phi_{j}(x, \lambda)\right| \leqslant \sqrt{2} \cosh \tau_{2} h \exp \left\{\frac{d \sinh T h}{T}\right\}, \quad j \in\{s, a\}
$$

(ii) Now we prove (A1) and (A2). Let $q \in C^{1}(\mathbb{R})$. From (35), by straightforward manipulations we have

$$
\Phi_{j}^{\prime}(x, \tau)-\mathrm{i} \tau \Phi_{j}(x, \tau)=\frac{\mathrm{i}}{\tau} p(x) \phi_{j}(x, \lambda)
$$

by multiplying the above equation by $\mathrm{e}^{-\mathrm{i} \tau x}$, integrating by parts twice and observing that

$$
\begin{aligned}
2 \int_{0}^{x} \mathrm{e}^{-\mathrm{i} \tau y} p(y) \phi_{j}(y, \lambda) \mathrm{d} y= & \int_{0}^{x} \mathrm{e}^{-\mathrm{i} \tau y} p(y) \Phi_{j}(y, \lambda) \mathrm{d} y+\frac{1}{\mathrm{i} \tau} \int_{0}^{x} \mathrm{e}^{-\mathrm{i} \tau y} p^{\prime}(y) \phi_{j}(y, \lambda) \mathrm{d} y \\
& -\left[\frac{\mathrm{e}^{-\mathrm{i} \tau y}}{\mathrm{i} \tau} p(y) \phi_{j}(y, \lambda)\right]_{y=0}^{y=x}
\end{aligned}
$$


it follows that $\Phi_{j}$ satisfies

$$
\begin{aligned}
\Phi_{j}(x, \tau) \mathrm{e}^{-\mathrm{i} \tau x}= & \Phi_{j}(0, \tau)+\frac{\mathrm{i}}{2 \tau} \int_{0}^{x} \mathrm{e}^{-\mathrm{i} \tau y} p(y) \Phi_{j}(y, \lambda) \mathrm{d} y \\
& +\frac{1}{2 \tau^{2}}\left\{\int_{0}^{x} \mathrm{e}^{-\mathrm{i} \tau y} p^{\prime}(y) \phi_{j}(y, \lambda) \mathrm{d} y-\mathrm{e}^{-\mathrm{i} \tau x} p(x) \phi_{j}(x, \lambda)+p(0) \phi_{j}(0, \lambda)\right\}
\end{aligned}
$$

By setting $M_{j}(x, \tau)=\sup _{s \in[0, x]}\left|\Phi_{j}(s, \lambda) \mathrm{e}^{-\mathrm{i} \tau s}\right|$ and from (A4), we get

$$
\begin{aligned}
M_{j}(x, \tau) \leqslant & \left|\Phi_{j}(0, \tau)\right|+\frac{1}{2|\tau|} \int_{0}^{x} p(y) M_{j}(y, \tau) \mathrm{d} y \\
& +\frac{1}{2|\tau|^{2}}\left\{C \int_{0}^{x} \mathrm{e}^{\tau_{2} y}\left|p^{\prime}(y)\right| \mathrm{d} y+C d^{2} \mathrm{e}^{\tau_{2} x}+p(0)\left|\phi_{j}(0, \lambda)\right|\right\}
\end{aligned}
$$

for $|\tau| \geqslant d$, where $C$ is the right-hand side of (A4). Thus, Gronwall's Lemma yields the following estimate for $M_{j}$ :

$$
\begin{aligned}
M_{j}(x, \tau) \leqslant & {\left[\left|\Phi_{j}(0, \tau)\right|+\frac{C d^{2}}{2|\tau|^{2}}+p(0)\left|\phi_{j}(0, \lambda)\right|\right] \exp \left\{\frac{1}{2|\tau|} \int_{0}^{x} p(y) \mathrm{d} y\right\} } \\
& +\frac{C}{2|\tau|^{2}} \int_{0}^{x} \exp \left\{\frac{1}{2|\tau|} \int_{s}^{x} p(y) \mathrm{d} y\right\} \mathrm{e}^{\tau_{2} s}\left[\left|p^{\prime}(s)\right|+\tau_{2} d^{2}\right] \mathrm{d} s
\end{aligned}
$$

since $\Phi_{s}(0, \tau)=1, \Phi_{a}(0, \tau)=\sqrt{\tau^{2}+d^{2}} / \mathrm{i} \tau$ and $0 \leqslant p(x) \leqslant d^{2}$, we have

$$
M_{j}(x, \tau) \leqslant \mathrm{e}^{d h / 2}\left\{1+\sqrt{2}+\frac{C}{2 d^{2}} \mathrm{e}^{T x}\left[d^{2}+|q|_{B V}\right]\right\}
$$

for $|\tau| \geqslant d$. By a standard approximation argument we can infer that (A7) holds for every $q$ of bounded variation. By (A6), (A4) and (A7), we get that

$$
\Phi_{j}(x, \tau)=\Phi_{j}(0, \tau) \mathrm{e}^{\mathrm{i} \tau x}+\mathcal{O}\left(\frac{1}{|\tau|}\right)
$$

Again, from (A6) and the above asymptotic formula, we obtain (A1) and (A2).

In Lemmas A.2 and A.3, we will use the following inequality:

$$
|\operatorname{Im} \sin t| \leqslant \max \left\{1, \frac{\sqrt{d^{2}-\gamma_{M}}}{2 \beta_{0}}\right\}, \quad t \in \mathscr{C}_{\vartheta}, \vartheta \in[0, \pi / 2]
$$

\section{Lemma A.2}

Let $g$ be defined by (39). Then, the following asymptotic expansion

$$
g(x, \xi ; t)=\frac{1}{4 \pi i} \mathrm{e}^{\mathrm{i} \beta_{0}\left(\{x\}_{h}-\xi\right) \sin t}\left[1+\frac{\mathrm{i}}{2 \beta_{0} \sin t} \int_{\{\xi\}_{h}}^{\{x\}_{h}} p(y) \mathrm{d} y\right]+\mathcal{O}\left(\frac{1}{|\sin t|^{2}}\right)
$$

holds uniformly as $t \rightarrow \infty$ on $\mathscr{C}_{\vartheta}$ for $\vartheta \in[0, \pi / 2], x \in \mathbb{R}$ and $\xi$ bounded. 


\section{G. CIRAOLO AND R. MAGNANINI}

Proof

The proof is a straightforward consequence of Lemma A.1 and (A8) and hence is omitted.

Lemma A.3

Let $g$ be given by (39). Then $g$ is a bounded function of $x, \xi \in \mathbb{R}$, if $\xi$ is bounded, and $t \in \mathscr{C}_{\vartheta}$, $\vartheta \in[0, \pi / 2]$.

\section{Proof}

(i) First, we prove an estimate for $\phi_{j}\left(x, \tau^{2}+d^{2}\right)$ for $|\tau| \leqslant d,|\operatorname{Im} \tau| \leqslant T$ and $|x| \leqslant h$. By setting $\lambda=\tau^{2}+d^{2}$ and $\eta_{j}(x, \lambda)=\sup _{s \in[0, x]}\left|\phi_{j}(s, \lambda)\right|$ as before, from (A3) and since $|\sin \tau x / \tau x|$ is bounded by the constant $B=\sqrt{\cosh ^{2}(T h)+\sinh ^{2}(T h) /(T h)^{2}}$, we have

$$
\begin{aligned}
& \eta_{s}(x, \lambda) \leqslant \cosh (T x)+B \int_{0}^{|x|} p(y)|x-y| \eta_{s}(y, \lambda) \mathrm{d} y \\
& \eta_{a}(x, \lambda) \leqslant B\left\{\sqrt{2} d|x|+\int_{0}^{|x|} p(y)|x-y| \eta_{a}(y, \lambda) \mathrm{d} y\right\}
\end{aligned}
$$

Gronwall's Lemma yields

$$
\left|\phi_{j}(x, \lambda)\right| \leqslant \min \{\cosh (T h), \sqrt{2} d h B\} \exp \left(B \frac{d^{2} h^{2}}{2}\right)
$$

for $|x| \leqslant h,|\tau| \leqslant d$ and $|\operatorname{Im} \tau| \leqslant T$.

(ii) To complete the proof, we note that from (A5) it follows that

$$
\left|\frac{\Phi_{j}(x, \tau)}{\Phi_{j}(h, \tau)}\right| \leqslant \frac{d \mathrm{e}^{T h}}{\left|\tau \Phi_{j}(h, \tau)\right|}\left(\sqrt{2}+d h \mathrm{e}^{T h} \sup _{x \in[-h, h]}\left|\phi_{j}(x, \lambda)\right|\right)
$$

and since $\tau \Phi_{j}(h, \tau) \neq 0$ far from the poles of $g$, we have that $\Phi_{j}(x, \tau) / \Phi_{j}(h, \tau)$ is bounded for $\tau=\beta_{0} \sin t, t \in \mathscr{C}_{\vartheta}, \vartheta \in[0, \pi / 2]$ and for $|\tau| \leqslant d$. Thus, the assertion of the lemma follows from (A10) and Lemma A.2.

\section{ACKNOWLEDGEMENTS}

The authors wish to thank the anonymous referees for their helpful comments and suggestions.

This work has been partially supported by the PRIN project 'Equazioni alle derivate parziali e disuguaglianze funzionali: aspetti quantitativi, proprietà geometriche e qualitative, applicazioni’, financed by MIUR.

\section{REFERENCES}

1. Sommerfeld A. Die Greensche Funktion der Schwingungsgleichung. Jahresber Deutschen Mathematiker Vereinigung 1912; 21:309-353.

2. Sommerfeld A. Partial Differential Equations in Physics. Academic Press: New York, 1949.

3. Magnus W. Über Eindeutigkeitsfragen bei einer Randwertaufgabe von $\Delta u+k^{2} u=0$. Jahresber Deutschen Mathematiker Vereinigung 1942; 52:177-188.

4. Magnus W. Fragen der Eindeutigkeit und des Verhaltens im Unendlichen für Lösungen von $\Delta u+k^{2} u=0$. Abhandlungen aus dem Mathematischen Seminar der Universität Hamburg 1949; 16:77-94. 


\section{UNIQUENESS FOR 2-D WAVEGUIDES}

5. Rellich F. Über das asymptotische Verhalten del Lösungen von $\Delta u+\lambda u=0$ in unendlichen Gebieten. Jahresber Deutschen Mathematiker Vereinigung 1943; 53:57-65.

6. Miranker WL. Uniqueness and representation theorems for solutions of $\Delta u+k^{2} u=0$ in infinite domains. Journal of Mathematics and Mechanics 1957; 6:847-854.

7. Schot SH. Eighty years of Sommerfeld's radiation condition. Historia Mathematica 1992; 19:385-401.

8. Miranker WL. The reduced wave equation in a medium with a variable index of refraction. Communications on Pure and Applied Mathematics 1957; 10:491-502.

9. Eidus D. Radiation principle for inhomogeneous media. Journal of Mathematical Physics 1998; 39(3):1458-1477.

10. Hörmander L. The Analysis of Linear Partial Differential Operators. II. Differential Operators with Constant Coefficients. Classics in Mathematics. Springer: Berlin, 2005.

11. Agmon S, Hörmander L. Asymptotic properties of solutions of differential equations with simple characteristics. Journal d Analyse Mathematique 1976; 30:1-38.

12. Vainberg BR. Asymptotic Methods in Equations of Mathematical Physics. Gordon and Breach: New York, 1989.

13. Schulenberger JR, Wilcox $\mathrm{CH}$. The limiting absorption principle and spectral theory for steady-state wave propagation in inhomogeneous anisotropic media. Archive for Rational Mechanics and Analysis 1971; 41:46-65.

14. Wilcox CH. Sound Propagation in Stratified Fluids. Applied Mathematical Sciences, vol. 50. Springer: New York, 1984.

15. Weder R. Spectral and Scattering Theory for Wave Propagation in Perturbed Stratified Media. Applied Mathematical Sciences, vol. 87. Springer: New York, 1991.

16. Ben-Artzi M, Dermenjian Y, Monsef A. Resolvent kernel estimates near thresholds. Differential and Integral Equations 2006; 19(1):1-14.

17. Zhang B. On transmission problems for wave propagation in two locally perturbed half spaces. Mathematical Proceedings of the Cambridge Philosophical Society 1994; 115:545-558.

18. Perthame B, Vega L. Energy concentration and Sommerfeld condition for Helmholtz and Liouville equations. Comptes Rendus de l'Academie des Sciences de Paris, Serie Mathematique 2003; 337(9):587-592.

19. Peters AS, Stoker JJ. A uniqueness theorem and a new solution for Sommerfeld's and other diffraction problems. Communications on Pure and Applied Mathematics 1954; 7:565-585.

20. Snyder AW, Love D. Optical Waveguide Theory. Chapman \& Hall: London, 1974.

21. Jäger W, Saitō Y. On the spectrum of the reduced wave operator with cylindrical discontinuity. Forum Mathematicum 1997; 9:29-60.

22. Jäger W, Saitō Y. The reduced wave equation in layered materials. Osaka Journal of Mathematics 1997; 34(2):267-301.

23. Reichardt H. Ausstrahlungsbedingungen fur die wellengleihung. Abhandlungen aus dem Mathematischen Seminar der Universität Hamburg 1960; 24:41-53.

24. Nosich AI. Radiation conditions, limiting absorption principle, and general relations in open waveguide scattering. Journal of Electromagnetic Waves and Applications 1994; 8(3):329-353.

25. Nosich AI, Shestopalov VP. Radiation conditions and uniqueness theorems for open waveguides. Soviet Journal of Communications Technology and Electronics 1989; 34:107-115.

26. Kartchevski EM, Nosich AI, Hanson GW. Mathematical analysis of the generalized natural modes of an inhomogeneous optical fiber. SIAM Journal on Applied Mathematics 2005; 65(6):2033-2048.

27. $\mathrm{Xu}$ Y. Scattering of acoustic waves by an obstacle in a stratified medium. Partial Differential Equations with Real Analysis. Pitman Research Notes in Mathematics Series, vol. 263, England. 1992; 147-168.

28. $\mathrm{Xu}$ Y. Radiation condition and scattering problem for time-harmonic acoustic waves in a stratified medium with a nonstratified inhomogeneity. IMA Journal of Applied Mathematics 1995; 54(1):9-29.

29. Xu Y. Reciprocity relations and completeness of far-field pattern vectors for obstacle scattering of acoustic wave in a stratified medium. Mathematical Methods in the Applied Sciences 1995; 18(1):51-66.

30. Magnanini R, Santosa F. Wave propagation in a 2-D optical waveguide. SIAM Journal on Applied Mathematics 2001; 61:1237-1252

31. Ciraolo G. A boundary variation method for waveguide couplers. Applicable Analysis, accepted for publication.

32. Wilcox C. Spectral analysis of the Pekeris operator in the theory of acoustic wave propagation in shallow water. Archive for Rational Mechanics and Analysis 1975/1976; 60(3):259-300.

33. DeSanto JA. Scalar Wave Theory. Springer: Berlin, 1992.

34. Titchmarsh EC. Eigenfunction Expansions Associated with Second-order Differential Equations. Clarendon Press: Oxford, 1946.

35. Ciraolo G. Non-rectilinear waveguides: analytical and numerical results based on the Green's function. Ph.D. Thesis, 2006. Available from: http://www.math.unifi.it/ ciraolo/. 


\section{G. CIRAOLO AND R. MAGNANINI}

36. Coddington EA, Levinson N. Theory of Ordinary Differential Equations. McGraw-Hill: New York, 1955.

37. Agmon S. Spectral properties of Schrödinger operators and scattering theory. Annali della Scuola Normale Superiore di Pisa, Classe di Scienze, Scuola Normale Superiore, Serie IV 1975; 2:151-218.

38. Leis R. Initial Boundary Value Problems in Mathematical Physics. Wiley: New York, 1986.

39. Ciraolo G, Magnanini R. Analytical results for 2-D non-rectilinear waveguides based on the Green's function. Mathematical Methods in the Applied Sciences 2008; 31(13):1587-1606.

40. Adams RA, Fournier JG. Sobolev Spaces. Academic Press: New York, 2003.

41. Gilbarg D, Trudinger NS. Elliptic Partial Differential Equations of Second Order. Springer: Berlin, 1983.

42. Bender C, Orszag SA. Advanced Mathematical Methods for Scientists and Engineers. McGraw-Hill: New York, 1978.

43. Zettl A. Sturm-Liouville Theory. Mathematical Surveys and Monographs, vol. 121. American Mathematical Society: Providence, RI, 2005.

44. Sansone G, Conti R. Non-linear Differential Equations. Pergamon Press: Oxford, 1964. 